\title{
Would Repurposing Minocycline Alleviate Neurologic Manifestations of COVID-19?
}

\author{
Aline C. Oliveira ${ }^{1}$, Elaine M. Richards ${ }^{1}$, Marianthi M. Karas ${ }^{1}$, Carl J. Pepine ${ }^{2}$ and \\ Mohan K. Raizada ${ }^{1 *}$ \\ ${ }^{1}$ Department of Physiology and Functional Genomics, College of Medicine, University of Florida, Gainesville, FL, \\ United States, ${ }^{2}$ Division of Cardiovascular Medicine, Department of Medicine, College of Medicine, University of Florida, \\ Gainesville, FL, United States
}

Keywords: neuroinflammation, SARS-CoV-2, minocycline, respiratory syndrome, autonomic system, microglia, COVID-19, hypoxia

\section{INTRODUCTION}

Severe acute respiratory syndrome coronavirus-2 (SARS-CoV-2) is the etiologic agent of COVID19 pandemic (Zhu et al., 2020). SARS-CoV-2 causes systemic infection varying in severity from asymptomatic, to mild (fever, cough, loss of smell and taste, leg pain, headache, diarrhea, fatigue), to multi-organ dysfunction/failure (Chen N. et al., 2020). Acute lung injury is the hallmark of COVID-

OPEN ACCESS

Edited by:

Jacob Raber,

Oregon Health and Science University, United States

Reviewed by: Lucio Tremolizzo, University of Milano-Bicocca, Italy Doo-Sup Choi, Mayo Clinic College of Medicine \& Science, United States

*Correspondence: Mohan K. Raizada mraizada@ufl.edu

Specialty section: This article was submitted to Neuropharmacology,

a section of the journal

Frontiers in Neuroscience

Received: 29 June 2020

Accepted: 27 August 2020 Published: 30 September 2020

Citation:

Oliveira AC, Richards EM, Karas MM,

Pepine CJ and Raizada MK (2020) Would Repurposing Minocycline Alleviate Neurologic Manifestations of

COVID-19?

Front. Neurosci. 14:577780

doi: 10.3389/fnins.2020.577780
19 and a subset of patients develop pneumonia and severe dyspnea requiring ICU admission. Most patients in critical condition have advanced age and pre-existing hypertension, cardiovascular disease (CVD), diabetes, obesity, and/or chronic lung conditions (Chen N. et al., 2020; Sharma et al., 2020b). Dyspnea and saturation of $90 \%$ or less despite oxygen supplementation is a major risk factor for fatal outcomes (Xie et al., 2020).

Angiotensin 1 converting enzyme 2 (ACE2) is the receptor that SARS-CoV-2 uses to enter host cells. ACE2 is highly expressed in lung but widespread distribution of ACE2 provides potential for infection of other organs. ACE2 is a member of the renin-angiotensin system (RAS) that plays an important role in the homeostasis of the cardiopulmonary system. The RAS consists of a vasoconstrictive, pro-inflammatory, and pro-fibrotic axis that includes angiotensin converting enzyme, angiotensin II and angiotensin type 1 receptor. Actions of this axis are balanced by the vasoprotective axis of the RAS that includes ACE2, angiotensin-(1-7) and MAS). Protective effects of ACE2 against cardiorespiratory diseases have been explored for 20 years (Sharma et al., 2020b).

After binding SARS-CoV-2, ACE2 is endocytosed downregulating cell surface ACE2. This explains the systemic RAS imbalance and increased pro-inflammatory Ang II observed in COVID19 that intensifies multi-organ damage (Liu et al., 2020). Similarly, while ACE2-knock out mice develop severe acute respiratory distress, mice overexpressing ACE2 develop neither pulmonary hypertension $(\mathrm{PH})$ nor neuroinflammation in a chronic hypoxia model. This illustrates the critical importance of ACE2 in balancing protective and pro-inflammatory effectors of RAS, by converting pro-inflammatory Ang II into beneficial Ang-(1-7). A randomized clinical trial evaluating efficacy, safety, and clinical impact of intravenous Ang-(1-7) vs. standard treatment in ICU patients with COVID-19 is underway (NCT04332666). Rationale for targeting the RAS to minimize systemic manifestations of COVID-19 are discussed in detail elsewhere (Sharma et al., 2020b).

\section{SARS-COV-2 AND THE CENTRAL NERVOUS SYSTEM}

It is becoming increasingly evident that COVID-19 also affects the central nervous system (AsadiPooya and Simani, 2020; Chu et al., 2020; Kanberg et al., 2020; Li et al., 2020; Mao et al., 2020; Moriguchi et al., 2020; Reichard et al., 2020; Sellner et al., 2020; Solomon et al., 2020; Yashavantha Rao and Jayabaskaran, 2020). However, controversial results regarding direct or indirect effects 
of SARS-COV-2 in the brain highlight the complexity of this disease and may indicate unique responses in individuals with no discernible common pattern yet uncovered. In vitro studies show that SARS-CoV-2 not only infects, but also replicates in neurons, a phenomenon not observed during SARS-CoV infection (Chu et al., 2020). ACE2 is expressed throughout the CNS, in neurons, glial, endothelial, and arterial smooth muscle cells (Xia and Lazartigues, 2008), allowing virus to infect the brain and impair inter-and intra-cellular communication. An observational study of 214 COVID-19 patients reported 78 patients exhibiting three categories of neurologic manifestation: CNS effects such as dizziness, headache, impaired consciousness (somnolence, stupor, and coma), confusion and delirium in conscious patients, acute cerebrovascular disease, ataxia, seizures; peripheral nervous system effects such as taste, smell or vision impairments, nerve pain, and musculoskeletal injury (Mao et al., 2020). Among 64 consecutive hospitalized COVID-19 patients, 58 exhibited neurological symptoms (encephalopathy, agitation, confusion, and corticospinal tract signs) (Helms et al., 2020a). A recent MRI-based 3-month follow-up study demonstrates that $55 \%$ of patients who recovered from COVID-19 infection had neurological symptoms and exhibited significantly higher gray matter area in brain regions relevant to memory, smell, tremor etc. (Yiping et al., 2020). Together, these data indicate that neurological manifestations of COVID-19 are relatively frequent especially in patients with severe disease, raising questions about mechanism, chronic consequences, and management since neither a vaccine nor specific treatment is available. Evidence supports many routes for CNS effects of SARS-CoV-2. A common sign of SARS-CoV-2 infection is anosmia, resulting from infection of nasal epithelium and olfactory nerve damage. The virus may infect the CNS from this peripheral nerve by crossing synapses (Li et al., 2020) suggesting a peripheral to CNS route of viral transmission and damage. While one study showed that SARS-CoV-2 was not present in cerebrospinal fluid (CSF) of 7 patients with confirmed COVID-19 and neurological symptoms, another detected SARS-CoV-2 by RT-PCR in CSF of a 24 year-old man with meningitis/encephalitis (Moriguchi et al., 2020). This suggests viral entry from blood through the choroid plexus, but could also result by virus released from damaged cells in the brain into the CSF. More conclusively, post-mortem study of a 74 year-old man identified virus in the frontal lobe, with virallike particles in neuronal cell bodies, small vesicles of endothelial cells, and throughout the cerebral microvasculature into neural tissue. This suggests a hematogenous route as a likely pathway to the brain (Paniz-Mondolfi et al., 2020). While evidence for the influence of COVID-19 in the brain continues to emerge, longterm consequences of this effect remains under investigation.

Nonetheless, plasma biomarkers of neuronal injury and glial activation are positively correlated with severity of COVID19 symptoms (Kanberg et al., 2020) and were confirmed postmortem (Reichard et al., 2020). Regardless of viral expression in brain, acute hypoxic ischemic damage was detected in brains of all 18 patients with SARS-CoV-2 infection (Solomon et al., 2020) and may indicate another route to induce neuroinflammation and increase inflammatory cytokines. This resembles the effects of hypoxia in animal models, which are microglia activation, neuroinflammation, autonomic imbalance and increased inflammatory cytokines leading, over time, to pulmonary hypertension (Savale et al., 2009; Oliveira et al., 2018; Sharma et al., 2020a).

In addition, patients with severe infection have increased inflammatory responses with CNS-related symptoms as the main form of neurologic injury, indicating indirect effects of SARS-CoV-2 infection in the brain, via pulmonary disease with increased pro-inflammatory cytokines (Mao et al., 2020). Comparing 113 deceased with 161 recovered patients revealed increased interleukin (IL) 2 receptor, IL-6, IL-8, IL10 , and tumor necrosis factor (TNF) and $20 \%$ with hypoxic encephalopathy amongst the deceased (Chen T. et al., 2020). Elevated IL-6 and abnormal permeability of blood meningeal barrier associated with encephalopathy was identified in another cohort with 140 patients admitted to ICU with COVID-19 diagnosis. Eighty-four percent showed abnormal neurological examination and/or delirium with significant worse prognosis compared to patients without delirium and normal neurological exam (Helms et al., 2020b). It might be explained by the intense systemic inflammatory response that leads to blood-brain barrier leakage and higher permeability to peripheral cytokines that exacerbate neuroinflammation and consequently, neurological damage (Cain et al., 2019). Bone marrow-derived cells may also contribute to neuroinflammation as demonstrated in hypertensive rat models. These cells migrate to the CNS and differentiate into microglia-like cells (Santisteban et al., 2015). Interestingly, bone marrow cells from ACE2 deficient mice promoted increases in inflammatory markers, which further contribute to neuroinflammation (Thatcher et al., 2012).

\section{MINOCYCLINE}

Minocycline is part of the family of tetracycline antibiotics that possess both antibiotic and anti-inflammatory properties. Minocycline has been safely used for more than 40 years which produces beneficial effects in diverse pathophysiological conditions and is emerging as a useful drug in providing neuroprotection as a result of its ability of pass through the blood brain barrier (Garrido-Mesa et al., 2013). Although the precise mechanism of minocycline actions remain elusive, it appears that effects on multiple enzyme systems and pathways could account for its diverse actions. It inhibits bacterial protein synthesis by preventing aminoacyl-tRNA attachment to the ribosomal acceptor and downregulates the nuclear factor- $-\kappa$ B pathway. In addition, its inhibitory effects on key enzymes like inducible nitric oxide synthase, matrix metalloproteases, phospholipase A2, protein tyrosine nitration, caspases, and other apoptotic molecules, p38 map kinase, poly[ADP] ribose polymerasel are linked with minocycline's anti-inflammatory, immunomodulatory, and neuroprotective actions (Garrido-Mesa et al., 2013). Minocycline is a highly lipophilic molecule; this property increases its half-life and allows excellent tissue penetration, and it easily crosses the BBB. Finally, minocycline inhibits HIV activation, proliferation 


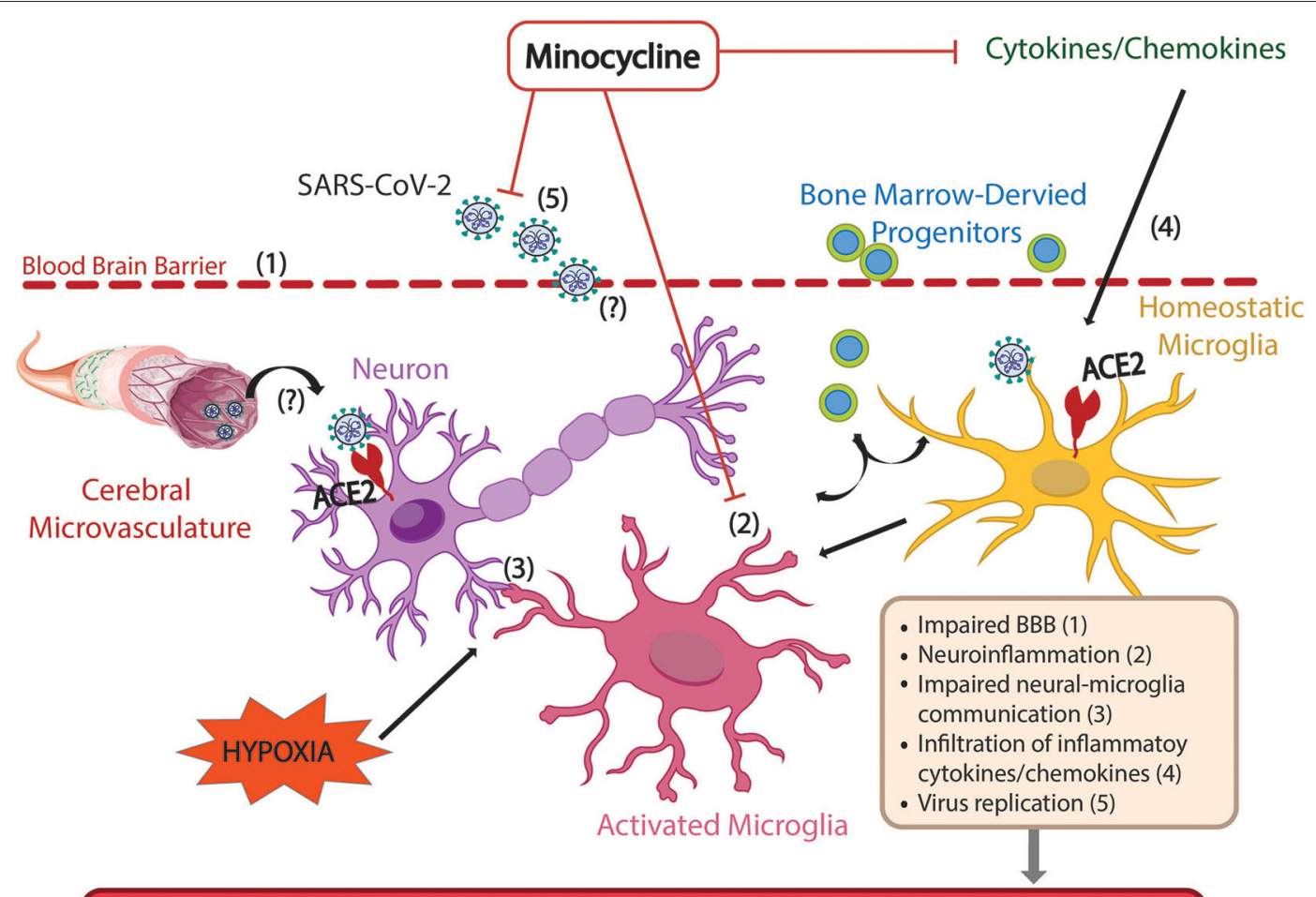

COVID-19: Neurological Manifestation

FIGURE 1 | Possible sites of action of minocycline for alleviation of neurological manifestations of Covid-19. Neurological manifestations have been extensively reported in COVID-19 patients, and are associated with more severe symptoms. These manifestations may arise by direct and/or indirect mechanisms following SARS-CoV-2 infection. The SARS-CoV-2 receptor, ACE2, is expressed in neurons, glial and endothelial cells allowing the virus to infect and spread in the brain, impairing cellular communication. SARS-CoV-2 virus has been demonstrated in brain parenchyma and possible routes for SARS-CoV-2 infiltration such as impaired blood brain barrier are shown. Anosmia, a common sign of SARS-CoV-2 infection reflecting olfactory nerve damage, may illustrate another possible route of virus infiltration, via peripheral nerves to the CNS. Indirect effects on CNS include those from Covid-19-induced hypoxia such as neuroinflammation, impaired neuron-microglia communication, autonomic imbalance, impaired BBB, increased inflammatory cytokines and increased release of BM-derived progenitor cells. This is consistent with experiments in rodents that directly investigated brain damage due to hypoxia. Minocycline, an anti-inflammatory antibiotic that readily penetrates the CNS, counteracts neuroinflammation, virus replication and attenuates the increase of pro-inflammatory cytokines. Together, these actions of minocycline alleviate hypoxia-induced neuroinflammation and impaired neural-microglial communication that may precipitate neuronal and glial injury, thus preventing potential long-term neurological consequences of COVID-19.

and replication in microglia, macrophages, and lymphocytes (Garrido-Mesa et al., 2013).

We recently found neuroinflammation critical in driving $\mathrm{PH}$ and impaired brain-lung-gut communication responsible for $\mathrm{PH}$-associated lung pathology; the number of activated microglia was positively correlated with $\mathrm{PH}$ severity (Oliveira et al., 2018; Sharma et al., 2018). This occurred in various $\mathrm{PH}$ models such as hypoxia, Sugen-hypoxia, and monocrotalineinduced $\mathrm{PH}$ in rats and mice. Moreover, we demonstrated that targeting neuroinflammation by inhibition of microglia activation could be a novel therapeutic strategy for $\mathrm{PH}$. First, absence of CX3CR1 expression, a condition rendering microglia unable to activate, prevented neuroinflammation and $\mathrm{PH}$ induced by hypoxia (Oliveira et al., 2018). Secondly, minocycline, an anti-inflammatory antibiotic also used as a microglial inhibitor, significantly reduced the numbers and activation of microglia in the paraventricular nucleus of hypothalamus (PVN), reduced expression of cytokines such as IL- $1 \beta$, IL-6, and TNF- $\alpha$, attenuated lung inflammation, heart hypertrophy and sympathetic drive in monocrotalinetreated rats (Sharma et al., 2018). Significant numbers of severely ill COVID-19 patients have diabetes and hypertension, comorbidities where neuroinflammation is an important contributor. Minocycline demonstrated beneficial outcomes in diabetes-linked peripheral and autonomic neuropathy and cognitive impairment in rodents (Syngle et al., 2014; Ismail et al., 2019; Mehta and Banerjee, 2019). Our study with a small group of diabetic, obese and hypertensive patients, with minocycline used on a compassionate basis, showed consistent weight loss, improved HbAlc, improved neuropathic pain and impressive decrease in blood pressure (Yellowlees Douglas et al., 2012). Data from our ongoing trial (NCT02133885) indicate that minocycline decreases blood pressure, active microglia and plasma pro-inflammatory cells in treatment-resistant hypertensive patients. These observations led us to propose the following hypothesis (Figure 1): Minocycline attenuates 
microglia activation and arrests neuro-inflammation resulting in restoration of normal neuronal-microglia communication, controlling the pro-inflammatory profile especially in moderate and severe COVID-19 patients. This decreases the risk of developing long-term CNS consequences of SARS-CoV-2 infection. The following evidence supports our hypothesis: (i) Minocycline is a safe anti-inflammatory drug; (ii) In addition to anti-microbial activity, it is anti-inflammatory, antioxidant, inhibits ion channels and apoptosis and promotes neuronal regeneration in rodents (Miyachi et al., 1986; Rifkin et al., 1994; Tikka et al., 2001; Liu et al., 2007; Nutile-McMenemy et al., 2007). These actions, in addition to its ability to inhibit microglia are likely to contribute to beneficial effects in inflammatory diseases and CNS disorders including stroke (O'Dell, 1999; Naderi et al., 2020); (iii) minocycline has high lipid solubility, readily crossing the $\mathrm{BBB}$; (iv) minocycline is the leading neuroprotective tetracycline, mainly by inhibition of microglia activation (Garrido-Mesa et al., 2013); (v) minocycline treatment effectively decreases inflammatory cytokines such as TNF, IL-1 $\beta$, and IL-6 (Sharma et al., 2018), all highly expressed in COVID-patients and related to increased neurological damage (Chen N. et al., 2020); (vi) it is not an antiviral, however, it might act directly on the coronavirus by chelating zinc compounds on matrix metalloproteinase necessary for virus survival, infiltration and replication in the host (Sodhi and Etminan, 2020); (vii) alterations in fecal microbiota in Covid-19 patients were associated with severity and fecal levels of SARS-CoV-2 and gut dysbiosis persisted even after recovery (Zuo et al., 2020). These might also be attenuated by minocycline since it has been shown to modulate the composition of the gut microbiota and attenuate gut pathology in hypertension and depression (Schmidtner et al., 2019; Sharma et al., 2019; Yang et al., 2020); (viii) Its ability to reduce lung inflammation in $\mathrm{PH}$ and potentially beneficial effects in diabetes, obesity and hypertension are added bonuses. Finally, COVID-19 is emerging as a complex disease involving multiple organs and patients undergo multiple drug treatment to control diverse symptoms. Therefore, potential drug interactions should be considered before addition of minocycline since this anti-inflammatory antibiotic could produce adverse interactions with anti-viral drugs, other antibiotics and drugs acting on the central nervous system.

\section{DISCUSSION}

The rapid increase in COVID-19 has led to recognition that neurological manifestations are more frequent than initially suspected with unknown long-term nervous system consequences at this early stage of the pandemic. Hundreds of registered clinical trials are currently evaluating the extension of neurological manifestations in COVID-19 patients, with some of those aiming to long-term effects on brain functions (e.g., ClinicalTrials.gov Identifier: NCT04401449). It will be imperative to better understand whether SARS-CoV-2 infection increases the incidence of, or predisposes to, neurological and neurodegenerative disorders like cognitive impairment and dementia. We need to understand whether the risk applies only to patients with neurological symptoms during initial infection or to all infected patients. This is of concern because while only $11 \%$ of rats infected with a related coronavirus exhibited neurological symptoms, $40 \%$ of animals without clinically recognized neurological signs had pathological brain lesions 8months after infection (Nagashima et al., 1979). This suggests that effects of SARS-CoV-2 infection on the CNS require very close attention, particularly because long-term CNS-effects may emerge in patients without neurological symptoms during initial infection. Therapies that could minimize these consequences would be very valuable.

In conclusion, we believe that not only is there is an urgent need for effective therapies to minimize systemic effects of SARSCoV-2 and its pro-inflammatory profile, but also to mitigate its CNS effects that are potentially long-term. Minocycline has effective mechanisms to protect the CNS, it attenuates neuroinflammation, counteracts the cytokine storm, modulates gut microbiome, and might inhibit viral replication. If confirmed, our hypothesis may represent an important contribution to prevent neurological impacts of infection and improve COVID19 management.

\section{AUTHOR CONTRIBUTIONS}

AO contributed substantially to the conception, design, and writing the opinion letter. ER contributed to the writing and provided critical revision. MK contributed to the final figure. $\mathrm{CP}$ revised it critically for important intellectual content. MR contributed the draft and revised it critically for important intellectual content. All authors read and approved the final manuscript.

\section{FUNDING}

This research was supported by NIH grants HL102033, HL110170, and AHA AWD07186.

\section{REFERENCES}

Asadi-Pooya, A. A., and Simani, L. (2020). Central nervous system manifestations of COVID-19: A systematic review. J. Neurol. Sci. 413:116832. doi: 10.1016/j.jns.2020.116832

Cain, M. D., Salimi, H., Diamond, M. S., and Klein, R. S. (2019). Mechanisms of pathogen invasion into the central nervous system. Neuron 103, 771-783. doi: 10.1016/j.neuron.2019.07.015

Chen, N., Zhou, M., Dong, X., Qu, J., Gong, F., Han, Y., et al. (2020). Epidemiological and clinical characteristics of 99 cases of 2019 novel coronavirus pneumonia in Wuhan, China: a descriptive study. Lancet 395, 507-513. doi: 10.1016/S0140-6736(20)30211-7

Chen, T., Wu, D., Chen, H., Yan, W., Yang, D., Chen, G., et al. (2020). Clinical characteristics of 113 deceased patients with coronavirus disease 2019: retrospective study. BMJ 368:m1091. doi: 10.1136/bmj. m1091 
Chu, H. C., Chan, J. F. W., Yuen, T. T. T., Shuai, H., Yuan, S., Wang, Y., et al. (2020). Comparative tropism, replication kinetics, and cell damage profiling of SARS-CoV-2 and SARS-CoV with implications for clinical manifestations, transmissibility, and laboratory studies of COVID-19: an observational study. Lancet Microbe 1, E14-E23. doi: 10.1016/S2666-5247(20)30004-5

Garrido-Mesa, N., Zarzuelo, A., and Gálvez, J. (2013). Minocycline: far beyond an antibiotic. Br. J. Pharmacol. 169, 337-352. doi: 10.1111/bph.12139

Helms, J., Kremer, S., Merdji, H., Clere-Jehl, R., Schenck, M., Kummerlen, C., et al. (2020a). Neurologic Features in Severe SARS-CoV-2 Infection. N. Engl. J. Med. 382, 2268-2270. doi: 10.1056/NEJMc2008597

Helms, J., Kremer, S., Merdji, H., Schenck, M., Severac, F., Clere-Jehl, R., et al. (2020b). Delirium and encephalopathy in severe COVID-19: a cohort analysis of ICU patients. Crit. Care 24:491. doi: 10.1186/s13054-020-03200-1

Ismail, C. A. N., Suppian, R., Aziz, C. B. A., and Long, I. (2019). Minocycline attenuates the development of diabetic neuropathy by modulating DREAM and BDNF protein expression in rat spinal cord. J. Diabetes Metab. Disord. 18, 181-190. doi: 10.1007/s40200-019-00411-4

Kanberg, N., Ashton, N. J., Andersson, L. M., Yilmaz, A., Lindh, M., Nilsson, S., et al. (2020). Neurochemical evidence of astrocytic and neuronal injury commonly found in COVID-19. Neurology. doi: 10.1212/WNL.0000000000010111. [Epub ahead of print].

Li, Y. C., Bai, W. Z., and Hashikawa, T. (2020). The neuroinvasive potential of SARS-CoV2 may play a role in the respiratory failure of COVID-19 patients. $J$. Med. Virol. 92, 552-555. doi: 10.1002/jmv.25728

Liu, Y., Yang, Y., Zhang, C., Huang, F., Wang, F., Yuan, J., et al. (2020). Clinical and biochemical indexes from 2019-nCoV infected patients linked to viral loads and lung injury. Sci. China Life Sci. 63, 364-374. doi: 10.1007/s11427-020$1643-8$

Liu, Z., Fan, Y., Won, S. J., Neumann, M., Hu, D., Zhou, L., et al. (2007). Chronic treatment with minocycline preserves adult new neurons and reduces functional impairment after focal cerebral ischemia. Stroke 38, 146-152. doi: 10.1161/01.STR.0000251791.64910.cd

Mao, L., Jin, H., Wang, M., Hu, Y., Chen, S., He, Q., et al. (2020). Neurologic manifestations of hospitalized patients with coronavirus disease 2019 in Wuhan, China. JAMA Neurol. 77, 683-690. doi: 10.1001/jamaneurol.2020.1127

Mehta, B. K., and Banerjee, S. (2019). Minocycline reverses diabetesassociated cognitive impairment in rats. Pharmacol. Rep. 71, 713-720. doi: 10.1016/j.pharep.2019.03.012

Miyachi, Y., Yoshioka, A., Imamura, S., and Niwa, Y. (1986). Effect of antibiotics on the generation of reactive oxygen species. J. Invest. Dermatol. 86, 449-453. doi: 10.1111/1523-1747.ep12285793

Moriguchi, T., Harii, N., Goto, J., Harada, D., Sugawara, H., Takamino, J., et al. (2020). A first case of meningitis/encephalitis associated with SARSCoronavirus-2. Int. J. Infect. Dis. 94, 55-58. doi: 10.1016/j.ijid.2020.03.062

Naderi, Y., Panahi, Y., Barreto, G. E., and Sahebkar, A. (2020). Neuroprotective effects of minocycline on focal cerebral ischemia injury: a systematic review. Neural Regen. Res. 15, 773-782. doi: 10.4103/1673-5374.268898

Nagashima, K., Wege, H., Meyermann, R., and ter Meulen, V. (1979). Demyelinating encephalomyelitis induced by a long-term corona virus infection in rats. A preliminary report. Acta Neuropathol. 45, 205-213. doi: $10.1007 /$ BF00702672

Nutile-McMenemy, N., Elfenbein, A., and Deleo, J. A. (2007). Minocycline decreases in vitro microglial motility, beta1-integrin, and Kv1.3 channel expression. J. Neurochem. 103, 2035-2046. doi: $10.1111 /$ j.1471-4159.2007.04889.x

O'Dell, J. R. (1999). Is there a role for antibiotics in the treatment of patients with rheumatoid arthritis? Drugs 57, 279-282. doi: 10.2165/00003495-199957030-00001

Oliveira, A. C., Sharma, R. K., Aquino, V., Lobaton, G., Bryant, A. J., Harrison, J. K., et al. (2018). Involvement of microglial cells in hypoxiainduced pulmonary hypertension. Am. J. Respir Cell Mol. Biol. 59, 271-273. doi: 10.1165/rcmb.2018-0042LE

Paniz-Mondolfi, A., Bryce, C., Grimes, Z., Gordon, R. E., Reidy, J., Lednicky, J., et al. (2020). Central Nervous system involvement by severe acute respiratory syndrome coronavirus-2 (SARS-CoV-2). J. Med. Virol. 92, 699-702. doi: 10.1002/jmv.25915

Reichard, R. R., Kashani, K. B., Boire, N. A., Constantopoulos, E., Guo, Y., and Lucchinetti, C. F. (2020). Neuropathology of COVID-19: a spectrum of vascular and acute disseminated encephalomyelitis (ADEM)-like pathology. Acta Neuropathol. 140, 1-6. doi: 10.1007/s00401-020-02166-2

Rifkin, B. R., Vernillo, A. T., Golub, L. M., Ramamurthy, N. S., (1994). Modulation of bone resorption by tetracyclines. Ann. N. Y. Acad. Sci. 732, 165-80. doi: 10.1111/j.1749-6632.1994.tb24733.x

Santisteban, M. M., Ahmari, N., Carvajal, J. M., Zingler, M. B., Qi, Y., Kim, S., et al. (2015). Involvement of bone marrow cells and neuroinflammation in hypertension. Circ. Res. 117, 178-191. doi: 10.1161/CIRCRESAHA.117.305853

Savale, L., Tu, L., Rideau, D., Izziki, M., Maitre, B., Adnot, S., et al. (2009). Impact of interleukin-6 on hypoxia-induced pulmonary hypertension and lung inflammation in mice. Respir Res. 10:6. doi: 10.1186/1465-9921-10-6

Schmidtner, A. K., Slattery, D. A., Gläsner, J., Hiergeist, A., Gryksa, K., Malik, V. A., et al. (2019). Minocycline alters behavior, microglia and the gut microbiome in a trait-anxiety-dependent manner. Transl. Psychiatry 9:223. doi: 10.1038/s41398-019-0556-9

Sellner, J., Taba, P., Öztürk, S., and Helbok, R. (2020). The need for neurologists in the care of COVID-19 patients. Eur. J. Neurol. 27, e31-e32. doi: $10.1111 /$ ene.14257

Sharma, R. K., Oliveira, A. C., Kim, S., Rigatto, K., Zubcevic, J., Rathinasabapathy, A., et al. (2018). Involvement of neuroinflammation in the pathogenesis of monocrotaline-induced pulmonary hypertension. Hypertension 71, 1156-1163. doi: 10.1161/HYPERTENSIONAHA.118.10934

Sharma, R. K., Oliveira, A. C., Yang, T., Karas, M. M., Li, J., Lobaton, G. O., et al. (2020a). Gut pathology and its rescue by ACE2 (angiotensin-converting enzyme 2) in hypoxia-induced pulmonary hypertension. Hypertension 76 , 206-216. doi: 10.1161/HYPERTENSIONAHA.120.14931

Sharma, R. K., Stevens, B. R., Obukhov, A. G., Grant, M. B., Oudit, G. Y., Li, Q., et al. (2020b). ACE2 (angiotensin-converting enzyme 2) in cardiopulmonary diseases: ramifications for the control of SARS-CoV-2. Hypertension 76, 651-661. doi: 10.1161/HYPERTENSIONAHA.120.15595

Sharma, R. K., Yang, T., Oliveira, A. C., Lobaton, G. O., Aquino, V., Kim, S., et al. (2019). Microglial cells impact gut microbiota and gut pathology in angiotensin II-induced hypertension. Circ. Res. 124, 727-736. doi: 10.1161/CIRCRESAHA.118.313882

Sodhi, M., and Etminan, M. (2020). Therapeutic potential for tetracyclines in the treatment of COVID-19. Pharmacotherapy 40, 487-488. doi: 10.1002/phar.2395

Solomon, I. H., Normandin, E., Bhattacharyya, S., Mukerji, S. S., Keller, K., Ali, A. S., et al. (2020). Neuropathological Features of Covid-19. N. Engl. J. Med. 383, 989-992. doi: 10.1056/NEJMc2019373

Syngle, A., Verma, I., Krishan, P., Garg, N., and Syngle, V. (2014). Minocycline improves peripheral and autonomic neuropathy in type 2 diabetes: MIND study. Neurol. Sci. 35, 1067-1073. doi: 10.1007/s10072-014-1647-2

Thatcher, S. E., Gupte, M., Hatch, N., and Cassis, L. A. (2012). Deficiency of ACE2 in bone-marrow-derived cells increases expression of TNF- $\alpha$ in adipose stromal cells and augments glucose intolerance in obese C57BL/6 Mice. Int. J. Hypertens 2012:762094. doi: 10.1155/2012/762094

Tikka, T., Usenius, T., Tenhunen, M., Keinänen, R., and Koistinaho, J. (2001). Tetracycline derivatives and ceftriaxone, a cephalosporin antibiotic, protect neurons against apoptosis induced by ionizing radiation. J. Neurochem. 78 , 1409-1414. doi: 10.1046/j.1471-4159.2001.00543.x

Xia, H., and Lazartigues, E. (2008). Angiotensin-converting enzyme 2 in the brain: properties and future directions. J. Neurochem. 107, 1482-1494. doi: 10.1111/j.1471-4159.2008.05723.x

Xie, J., Covassin, N., Fan, Z., Singh, P., Gao, W., Li, G., et al. (2020). Association between hypoxemia and mortality in patients with COVID-19. Mayo Clin. Proc. 95, 1138-1147. doi: 10.1016/j.mayocp.2020.04.006

Yang, T., Li, H., Oliveira, A. C., Goel, R., Richards, E. M., Pepine, C. J., et al. (2020). Transcriptomic signature of gut microbiome-contacting cells in colon of spontaneously hypertensive rats. Physiol. Genomics 52, 121-132. doi: 10.1152 physiolgenomics.00087.2019

Yashavantha Rao, H. C., and Jayabaskaran, C. (2020). The emergence of a novel coronavirus (SARS-CoV-2) disease and their neuroinvasive propensity may affect in COVID-19 patients. J. Med. Virol. 92, 786-790. doi: 10.1002/jmv.25918

Yellowlees Douglas, J., Bhatwadekar, A. D., Li Calzi, S., Shaw, L. C., Carnegie, D., Caballero, S., et al. (2012). Bone marrow-CNS connections: implications in the pathogenesis of diabetic retinopathy. Prog. Retin. Eye Res. 31, 481-494. doi: $10.1016 /$ j.preteyeres.2012.04.005 
Yiping, L. U., Li, X., Geng, D., Mei, N., Wu, P.-Y., Huang, C.-C., et al. (2020). Cerebral micro-structural changes in COVID-19 patients - an MRI-based 3month follow-up study. Lancet 25, 100484. doi: 10.1016/j.eclinm.2020.100484

Zhu, N., Zhang, D., Wang, W., Li, X., Yang, B., Song, J., et al. (2020). A novel coronavirus from patients with pneumonia in China, 2019. N. Engl. J. Med. 382, 727-733. doi: 10.1056/NEJMoa20 01017

Zuo, T., Zhang, F., Lui, G. C. Y., Yeoh, Y. K., Li, A. Y. L., Zhan, H., et al. (2020). Alterations in gut microbiota of patients with COVID19 during time of hospitalization. Gastroenterology, 159, 944-955.e8. doi: 10.1053/j.gastro.2020.05.048
Conflict of Interest: The authors declare that the research was conducted in the absence of any commercial or financial relationships that could be construed as a potential conflict of interest.

Copyright $\odot 2020$ Oliveira, Richards, Karas, Pepine and Raizada. This is an openaccess article distributed under the terms of the Creative Commons Attribution License (CC BY). The use, distribution or reproduction in other forums is permitted, provided the original author(s) and the copyright owner(s) are credited and that the original publication in this journal is cited, in accordance with accepted academic practice. No use, distribution or reproduction is permitted which does not comply with these terms. 